\title{
Easy and pleasant learning concept in optical design
}

\author{
Ilya Mimorov ${ }^{1}$, Vladimir Vasiliev ${ }^{2}$, Irina Livshits ${ }^{3}$, Roman Anitropov ${ }^{4}$ \\ ${ }^{1}$ Ilya Mimorov, Kotina street 6-189, 198332 St.-Petersburg, Russian Federation \\ ${ }^{1}$ I.Mimorov@iods.pro, ${ }^{2}$ Vasiliev@ mail.ifmo.ru , ${ }^{3}$ ecenter-optica10@yandex.ru, \\ roman_aspherik@yandex.ru
}

\begin{abstract}
.
This is important to understand and take into account when creating new educational programs. ICT plays an important role in this process because current generation is already accustomed to use it in everyday life. "Written" lectures, tutorials and books are much less popular than internet resources. This is why we propose a new concept of easy and pleasant learning in the field of optical design. Currently this is a complicated system of courses where it is taught how to start optical design, optimize it, calculate the tolerances, evaluate system quality, etc. In present publication we describe a concept on how to make an education process easy for understanding and pleasant to use for student of optical specializations.
\end{abstract}

Keywords: distance teaching, remote learning, i-learning, e-teaching, webinar, teaching knowledge base, software open source, internet, optics

\section{Introduction}

Knowledge - it is a unique and valuable experience gained from the development of science and humanity. Transfer of knowledge - not only a complex and delicate process that involves a number of difficulties, but also an important element in the life of any person. The process of exchange of knowledge can take many forms and systematized in different ways.

The development of modern society leads to the fact that knowledge is a necessary commodity, without which modern man is not possible to realize in the labor market. In this case, for knowledge is not given to the systematic regular time. Acquisition of knowledge should not interrupt the modern man from his current activities, in addition, it should be convenient and comfortable.

Modern software systems, which are used in the learning process, significantly improved options for flexible maintaining the necessary functionality to the customer. As the modern software systems for the calculation of the parameters or system design significantly transformed and has the first signs to be used on "thin" clients that means to run programs using internet connection and without installing them. It becomes very convenient and practical.

These information systems that support the educational process or the system of distance education have new opportunities with using current news and information from around the globe.

\section{Modern mistakes}

There are a lot of educational portals and databases for self-learning and only 
few of them became popular. If we look closer we understand that these projects are famous because of avoiding the main mistakes during the planning period before the development and implementation.

Many who are interested in the transfer of knowledge, after running web project are surprised that the influx of people wanting to get this or that information, take online courses, small enough or close to zero. Project leaders believe that the launch of a new project using advanced technology will be enough to let people know about the system. Lack of marketing, search, and attracting new partners and the active promotion of a new product leads to the fact that the project simply unknown and in the future, this unpopularity of the risk that developers and people involved in the project themselves lose interest in him.

The second main deterrent is badly configured interface, which makes the potential user desire to quickly close the page and never visit it. Still, the error in the design of the design and location of functional windows are the main factors that determine the usability of the system.

If the first impression of the internet users produces positive emotions, then the complication of the way information is stored and difficult search, the desire to use the system also significantly reduced. Ergonomics in design, simplicity and accessibility of the information required is a significant plus.

The problem inherent in the various methods of information storage is duplication. When looking at the wellknown search engines on the results of the query issued a huge number of options, which provide interesting information, which reduces the probability that a query to the correct site with the required content.

Active and passive learning is, in most already offer the creation of user profiles, in which a person can keep track of the courses or to schedule a future or authorized to make changes of own registered account at distant teaching system. It is not enough to attract people on a permanent basis. System must provide more functionality allow communicating between each other students and teachers.

Courses or knowledge system must support at least two languages, because at any moment students from other country can became an attendee to these courses or receive access to the knowledge base. This is necessary point which must be complied by teachers in all conditions.

\section{Teaching process points}

Easy and pleasant learning are the main targets in the distance teaching systems. During the teaching period system must match certain conditions:

- Monitoring of the quality of education

- Improving the quality of educational content

- Maintenance of the educational process

One of the goals of the educational process is to control the passage of student education program, and the value of distance technologies increases due to the introduction in the educational process of the individual educational trajectories. The listener now he controls his education and support system of the educational process is designed to facilitate his passage and point to specific gaps or 
weaknesses in the assimilation of the material.

The use of modern interactive technologies will improve the quality of the material provided to the listener. Should take into account the latest trends in the market in which the listener affects the quality of educational content, conducting its ongoing evaluation. Thus it became common practice for universities to improve the quality of educational products to conduct surveys after passing course participants to identify the strengths and weaknesses of the product.

This problem is the main objective of the development of distance learning technologies. Modern integrative technologies help students and teachers, above all, to reduce the time for the theoretical part and devote more time to consolidate skills and competencies of the student. Platform support training activities should be doing training for the listener comfortable and convenient process of teaching for teachers.

\section{System targets}

As the distance teaching process is not static and the material and methods of teaching can be changed and during this process the influence on the students must be reduced. Distance teaching system must match several conditions to provide such functionality:

- Flexible environment that provide high level of education

- Material quality

- Control points

- Feedback

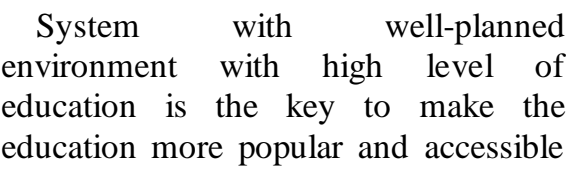

for the students. Its good advertisement for the system and the number of the attendee will grow up with high speed.

Well-designed system is only part of all the distance educational process and it is needed to teach the actual material to the students. The entire courses have to be reviewed at least once a year to make the correction and update the teaching material.

Good practice is to use the control points during the process. This could help to understand that material is actual, chosen correct method of education and the knowledge of students is equal to the planned education program.

Feedback links the people to estimate the functionality of the system after the course finished.

Fig. 1: Main flags of good distance teaching

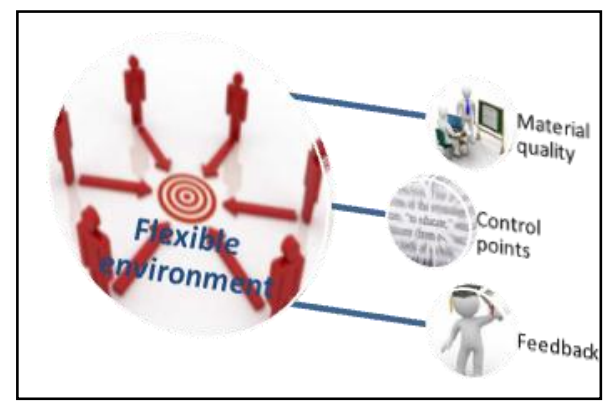

system.

Good planned process of distance education compliance such attributes can partially complement educational process making it easy and flexible.

\section{Material control}

To improve the quality of the teaching material teachers use control points and special metrics. After the control point is reached the feedback material is collected for analyzing also including the needed information from metrics. 
This step help the teaching personal identify the possible problems in the process of education, identify the mistakes in the material and collect the information for the future renovation. If the result of this step is not so good, teaching staff can introduce a change in the teaching program to retrieve the process of education and quality of the material to the normal and actual state.

\section{Summary}

It can be argued that optics is an important direction of development of a science. A lot of time takes the preparation courses of lectures and implementation special calculating of modeling optics program at the system of distance teaching. Professors of National Research University of Information Technologies, Mechanics and Optics obtain a big experience in teaching optical design using the modern distance teaching systems and ready to take part in building the new flexible distance teaching system that is suitable for usage in design of optics system and in search starting point. Consolidation of well-qualified technical specialist and professors with big experience will make the system more effective at teaching optics, with actual information and completely filled content.

Lecturer can't give the whole information during the webinar (mixed audio and video conference) and students have to read additional information in a free time and use the new information not only to make homework but to make a practical training independently.

\section{Acknowledgement}

Building of the test laboratory, research of the new methods of the distance education and help with filling distance teaching database is supported by NRU ITMO - National Research University Information Technologies Mechanics Optics.

\section{References}

[1] Mimorov Ilya, "Distance teaching in optical design", EET 2011.

[2] Mimorov Ilya, "Integration of a distance teaching system in an educational process", EMET 2012 